Objectives Prostate cancer is one of the most frequent cancers worldwide. Its aetiology is largely unknown with farming being suspected. Our aim was to identify occupational risk factors for prostate cancer in farmers in the French prospective cohort study AGRICAN.

Method During the period from enrollment (2005-2007) to 31 December 2009, 1664 incident prostate cancer cases were identified in the cohort $(n=92669)$ by linkage with cancer registries. Data on occupational history and agricultural exposures during lifetime on 13 types of crops and 5 types of animals were collected by the enrollment questionnaire. Hazard ratios (HR) were estimated using Cox regression analysis with attained age as underlying time scale.

Results Elevated risks were found for six agricultural activities: cattle, hogs, horses, grassland, wheat/barley and tobacco $(\mathrm{HR}=1.07$ to $1.16 ; \mathrm{p}=0.07$ to 0.23$)$. Prostate cancer risk was related to duration of work in wheat/barley and tobacco productions, number of cattle and hogs, and grassland area. We also observed an increased risk for fruit growing, with both duration and area. Increased prostate cancer risk was associated with applying pesticides on wheat/barley $(\mathrm{HR}=1.40, \mathrm{p}=0.06)$ with a duration relationship, and with making hay $(\mathrm{HR}=1.16, \mathrm{p}=0.03)$.

Conclusions Our analysis suggests that the risk of prostate cancer is increased in some farming activities, mainly in crops. This work will be completed by a multivariate analysis, with variables positively associated with the outcome in the previous analysis. The possible association between use of some chemical classes of pesticides and prostate cancer occurrence will be analysed through a crop-exposure matrix (PESTIMAT).

\section{RESPIRATORY HEALTH AFTER STARTING WORK: THE APPRENTICE STUDY}

${ }^{1}$ Kin Bong Hubert Lam, ${ }^{2}$ John Henderson, ${ }^{1} J o n$ G Ayres. ${ }^{1}$ University of Birmingham, Birmingham, UK; ${ }^{2}$ University of Bristol, Bristol, UK

\subsection{6/oemed-2014-102362.270}

Objectives Young adults between 16 and 24 years of age represent only $13 \%$ of the working population in the UK, yet they have higher rates of work-related accidents and injuries compared to workers of other age groups. However, little is known about the incidence and risk factors for occupational diseases such as occupational asthma in these individuals.

Method The study was based on data from the Avon Longitudinal Study of Parents and Children (ALSPAC). At 16 and 18 years, participants were asked to report their employment, along with questions on respiratory health.

Results At 16 years, those who left education and were employed had less severe asthma compared to those in education. At 18, asthma prevalence was higher among the employed, but the severity of asthma in these individuals did not differ significantly from those staying in education and who were not employed. There was no obvious relationship between the prevalence of asthma or the major occupational groups and the proportion of respondents reporting time lost from work.

Of all respondents who had asthma at 18 years, 3\% had lost time due to the condition, and $11 \%$ suggested the symptoms were better at weekends. Symptom improvement was mostly seen among waiters and waitresses, kitchen and catering assistants, and sales and retail assistants.

Conclusions The high prevalence of work-related symptoms suggests they may be substantial number of cases of occupational asthma in the young work force.
0209

SCREENING OF COMMON MENTAL DISORDERS AMONG OCCUPATIONAL GROUPS: A REVIEW OF DIMENSIONAL STRUCTURE OF SELF REPORTING QUESTIONNAIRE-SRQ20

${ }^{1}$ Kionna Bernardes, ${ }^{1}$ Fernando Carvalho, ${ }^{2}$ Tania Araujo. ${ }^{1}$ Federal University of Bahia, Salvador, Brazil; ${ }^{2}$ State University of Feira de Santana, Feira de Santana, Brazil

10.1136/oemed-2014-102362.271

Objectives The aim of this study was to evaluate the dimensional structure of the SRQ-20 in different occupational groups. It's a validation study that involved four sectional drawing surveys.

Method Individual analysis of the databases has been conducted by using the method of principal components. The latent class analysis has been used in the factor analysis of tetrachoric correlations in order to assess the dimensionality of the instrument, aiming to tailor the assessment method to the distribution of variable (categorical). The Vuong-Lo-Mendell-Rubin test adjusted Lo Mendell Rubin (LRT Test), the parametric bootstrap verisimilitude ratio test has been used to review the adequacy of the number of classes extracted in the recipiency of the CMD. The reliability of the latent classes has been evaluated by Latent Class Reliability Coefficient (LCRC).

Results The factor analysis of tetrachoric correlations allowed the assessment of the dimensions of the SRQ-20 and the comparison between different categories of workers. As a complement, the latent class analysis permitted the interpretation of subgroups for expressions of common mental disorders in the occupational sphere, providing in-depth research.

Conclusions The study combined two methods of analysis for assessing the dimensional structure of the SRQ 20 among workers. The factor analysis of tetrachoric correlations enabled us to verify the dimensional stability of the instrument, since the number of extracted factors and dimensions represented maintained similarities in the groups assessed. The study points to the use of measures of SRQ 20 as valid for screening TMC occupational groups.

\section{THE RELATIONSHIP BETWEEN NIGHT WORK AND MENTAL HEALTH AMONG HOSPITAL WORKERS}

Adenilda Arruda, Luciana Portela, Rosane Griep, Aline Silva-Costa, Lucia Rotenberg. Fundação Oswaldo Cruz, Rio de Janeiro/RJ, Brazil

\subsection{6/oemed-2014-102362.272}

Objectives Night work is suggested as a risk factor for mental health. The objectives of this study were (i) to test the association between the duration of exposure to night work and mental health, evaluated through depression and minor psychiatric disorder (MPD) and (ii) to assess the relationship between intention to leave the night shift and mental health.

Method A cross-sectional study was conducted among 432 workers (physical doctors and nursing personnel) at two hospitals in Manaus, Amazonas State, Brazil. Depression condition and MPD were assessed by PHQ-9 and SRQ-20, respectively. The statistical analyses (bivariate, multivariate logistic and multinomial regression) were conducted at SPSS (v.18.0).

Results Female workers, those younger than 38 years old, with children under the age of 6 showed higher prevalence of depression and MPD compared to their counterparts. No significant association was detected between time of exposure to night work and mental symptoms. After adjusting for age, sex, 